\title{
Identities of Generalized Fibonacci-Like Sequence
}

\author{
Mamta Singh $^{1}$, Omprakash Sikhwal ${ }^{2}$, Yogesh Kumar Gupta ${ }^{3, *}$ \\ ${ }^{1}$ Department of Mathematical Sciences and Computer application, Bhundelkhand University, Jhansi (U. P.) India \\ ${ }^{2}$ Department of Mathematics, Mandsaur Institute of Technology, Mandsaur (M. P.) India \\ ${ }^{3}$ Schools of Studies in Mathematics, Vikram University Ujjain, (M. P.) India \\ *Corresponding author: yogeshgupta.880@rediffmail.com
}

Received August 15, 2014; Revised September 16, 2014; Accepted October 08, 2014

\begin{abstract}
The Fibonacci and Lucas sequences are well-known examples of second order recurrence sequences. The Fibonacci sequence, Lucas numbers and their generalization have many interesting properties and applications to almost every field. Fibonacci sequence is defined by the recurrence formula $F_{n}=F_{n-1}+F_{n-2}, n \geq 2$ and $\mathrm{F}_{0}=0, \mathrm{~F}_{1}=1$, where $\mathrm{F}_{\mathrm{n}}$ is a ${ }^{\text {th }}$ number of sequence. Many authors have defined Fibonacci pattern based sequences which are popularized and known as Fibonacci-Like sequences. In this paper, Generalized Fibonacci-Like sequence is introduced and defined by the recurrence relation $M_{n}=M_{n-1}+M_{n-2}, n \geq 2$ with $M_{0}=2, M_{1}=s+1$, where $\mathrm{s}$ being a fixed integers. Some identities of Generalized Fibonacci-Like sequence are presented by Binet's formula. Also some determinant identities are discussed.
\end{abstract}

Keywords: Fibonacci sequence, Lucas Sequence, Generalized Fibonacci-Like Sequence, Binet's Formula

Cite This Article: Mamta Singh, Omprakash Sikhwal, and Yogesh Kumar Gupta, "Identities of Generalized Fibonacci-Like Sequence." Turkish Journal of Analysis and Number Theory, vol. 2, no. 5 (2014): 170-175. doi: 10.12691/tjant-2-5-3.

\section{Introduction}

Fibonacci numbers Fn and Lucas numbers Ln have delighted mathematicians and amateurs alike for centuries with their beauty and their propensity to pop up in quite unexpected places [3], [13] and [14]. It is well known that Fibonacci and Lucas numbers play an important role in many subjects such as algebra, geometry, and number theory. Their various elegant properties and wide applications have been studied by many authors.

The Fibonacci and Lucas sequences are examples of second order recursive sequences. The Fibonacci sequence [4] is defined by the recurrence relation:

$$
F_{n}=F_{n-1}+F_{n-2}, n \geq 2, \text { with } F_{0}=0, F_{1}=1 .
$$

The similar interpretation also exists for Lucas sequence. Lucas sequence [4] is defined by the recurrence relation:

$$
L_{n}=L_{n-1}+L_{n-2}, n \geq 2 \text { with } L_{0}=2, L_{1}=1 .
$$

Authors [1], [2] and [8] to [14] have been generalized second order recurrence sequences by preserving the recurrence relation and altering the first two terms of the sequence or preserving the first two terms of sequence and altering the recurrence relation slightly.

Horadam [1] introduced and studied properties of a generalized Fibonacci sequence $\left\{H_{n}\right\}$ and defined generalized Fibonacci sequence $\left\{H_{n}\right\}$ by the recurrence relation:

$$
H_{n+2}=H_{n+1}+H_{n}, H_{0}=q \text { and } H_{1}=p, n \geq 0,
$$

where $p, q$ are arbitrary integers.

Horadam [2] introduced and studied properties of another generalized Fibonacci sequence $\left\{w_{n}\right\}$ and defined generalized Fibonacci sequence $\left\{w_{n}\right\}$ by the recurrence relation:

$$
\begin{aligned}
& \left\{w_{n}\right\}=\left\{w_{n}(a, b ; p, q)\right\}: \mathrm{w}_{0}=a, \mathrm{w}_{1}=b, \\
& \mathrm{w}_{n}=p w_{n-1}-q w_{n-2}(\mathrm{n} \geq 2),
\end{aligned}
$$

where $a, b, p$ and $q$ are arbitrary integers.

Waddill and Sacks [10] extended the Fibonacci numbers recurrence relation and defined the sequence $\left\{P_{n}\right\}$ by recurrence relation:

$$
P_{n}=P_{n-1}+P_{n-2}+P_{n-3}, \quad n \geq 3,
$$

where $P_{0}, P_{1}$ and $P_{2}$ are not all zero given arbitrary algebraic integers.

Jaiswal [8] introduced and studied properties of generalized Fibonacci sequence $\left\{T_{n}\right\}$ and defined it by

$$
T_{n+1}=T_{n}+T_{n-1}, T_{1}=a \text { and } T_{2}=b, n \geq 1 .
$$

Falcon and Plaza [12] introduced $k^{\text {th }}$ Fibonacci sequence $\left\{F_{k, n}\right\}_{n \in N}$ and studied its properties. For any positive integer $k \geq 1, k^{\text {th }}$ Fibonacci sequence is defined by 


$$
\begin{aligned}
& F_{k, 0}=0, F_{k, 1}=1 \\
& \text { and } F_{k, \mathrm{n}+1},=k F_{k, \mathrm{n}}+F_{k, \mathrm{n}-1}, n \geq 1 .
\end{aligned}
$$

Many authors have been defined Fibonacci pattern based sequences which are known as Fibonacci-like sequences. The Fibonacci-Like sequence [4] is defined by recurrence relation,

$$
S_{n}=S_{n-1}+S_{n-2}, n \geq 2 \text { with } S_{0}=2, S_{1}=2 .
$$

The associated initial conditions $S_{0}$ and $S_{1}$ are the sum of initial conditions of Fibonacci and Lucas sequence respectively.

i.e. $S_{0}=F_{0}+L_{0}$ and $S_{1}=F_{1}+L_{1}$.

Fibonacci-Like sequence [6] is defined by the recurrence relation,

$$
H_{n}=2 H_{n-1}+H_{n-2} \text { for } n \geq 2 \text { with } H_{0}=2, H_{1}=1 \text {. }
$$

In this paper, Generalized Fibonacci-Like sequence is introduced. The Binet's formula is presented and established some identities of Generalized FibonacciLike sequence. Also determinants identities are discussed.

\section{Generalized Fibonacci-Like Sequence}

Generalized Fibonacci-Like sequence is introduced and defined by the recurrence relation

$$
M_{n}=M_{n-1}+M_{n-2}, n \geq 2 \text { with } M_{0}=2, M_{1}=s+1 \text {, }
$$

where $\mathrm{s}$ being a fixed integers. The first few terms are as follows:

$$
\begin{aligned}
& M_{0}=2, \\
& M_{1}=s+1, \\
& M_{2}=s+3, \\
& M_{3}=2 s+4, \\
& M_{4}=3 s+7, \\
& M_{5}=5 s+11, \\
& M_{6}=8 s+18, \\
& B_{7}=13 s+29 \text { and so on. }
\end{aligned}
$$

The characteristic equation of recurrence relation (2.1) is $t^{2}-t-1=0$. which has two real roots

$$
\alpha=\frac{1+\sqrt{5}}{2} \text { and } \beta=\frac{1-\sqrt{5}}{2} \text {. }
$$

Also, $\alpha \beta=-1, \alpha+\beta=1, \alpha-\beta=\sqrt{5}, \alpha^{2}+\beta^{2}=3$.

Generating function of generalized Fibonacci-Like sequence is

$$
\sum_{n=0}^{\infty} M_{n} t^{n}=M(t)=\frac{2+(s-1) t}{1-t-t^{2}} .
$$

Binet's formula of Generalized Fibonacci-Like sequence is defined by

$$
M_{n}=C_{1} \alpha^{n}+C_{2} \beta^{n}=C_{1}\left(\frac{1+\sqrt{5}}{2}\right)^{n}+C_{2}\left(\frac{1-\sqrt{5}}{2}\right)^{n}
$$

Here, $C_{1}=\frac{s+\sqrt{5}}{\sqrt{5}}$ and $C_{2}=\frac{\sqrt{5}-s}{\sqrt{5}}$. Also,

$$
\begin{aligned}
& C_{1} C_{2}=\frac{5-s^{2}}{(\alpha-\beta)^{2}}=\frac{5-s^{2}}{5}, C_{1} \beta+C_{2} \alpha=-s+1, \\
& C_{1} \alpha+C_{2} \beta=s+1, C_{1} \beta^{2}+C_{2} \alpha^{2}=3 \\
& C_{1}+C_{2}=M_{0}=2 .
\end{aligned}
$$

\section{Identities of Generalized Fibonacci- Like Sequence}

Now some identities of Generalized Fibonacci-Like sequence are present using generating function and Binet's formula. Authors [6,7] have been described such type identities.

Theorem (3.1). (Explicit Sum Formula) Let $M_{n}$ be the $n^{\text {th }}$ term of generalized Fibonacci-Like sequence. Then

$$
\left.M_{n}=2 \sum_{k=0}^{\left[\frac{n}{2}\right.}\right]\left(\begin{array}{c}
n-k \\
\mathrm{k}
\end{array}\right)+(s-1) \sum_{k=0}^{\left[\frac{n-1}{2}\right]}\left(\begin{array}{c}
n-k-1 \\
\mathrm{k}
\end{array}\right) \text {. }
$$

Proof. By generating function (2.3), we have

$$
\begin{aligned}
& \sum_{n=0}^{\infty} M_{n} t^{n}=M(t)=\frac{2+(s-1) t}{1-t-t^{2}}=\{2+(s-1) t\}\left(1-t-t^{2}\right)^{-1} \\
& =\{2+(s-1) t\}\left[1-\left(t+t^{2}\right)\right]^{-1} \\
& =\{2+(s-1) t\} \cdot \sum_{n=0}^{\infty}\left(t+t^{2}\right)^{n}=\{2+(s-1) t\} \cdot \sum_{n=0}^{\infty} t^{n}(1+t)^{n} \\
& =\{2+(s-1) t\} \cdot \sum_{n=0}^{\infty} t^{n} \sum_{k=0}^{n}\left(\begin{array}{l}
n \\
k
\end{array}\right)^{t} k \\
& =\{2+(s-1) t\} \sum_{n=0}^{\infty} \sum_{k=0}^{n} \frac{\underline{n}}{\underline{k} \underline{\underline{n}-k}} t^{n+k}
\end{aligned}
$$

(Replace $\mathrm{n}$ by $\mathrm{n}+\mathrm{k}$ )

$$
\begin{aligned}
& =\{2+(s-1) t\} \sum_{n=0}^{\infty} \sum_{k=0}^{\infty} \frac{\underline{\underline{n+k}}}{\underline{k} \underline{\underline{n}}} t^{n+2 k} \\
& =\{2+(s-1) t\} \sum_{n=0}^{\infty} \sum_{k=0}^{\left[\frac{n}{2}\right]} \frac{\underline{n-k}}{\underline{k}\lfloor n-2 k} t^{n}
\end{aligned}
$$

(Replace $\mathrm{n}$ by $\mathrm{n}-2 \mathrm{k}$ )

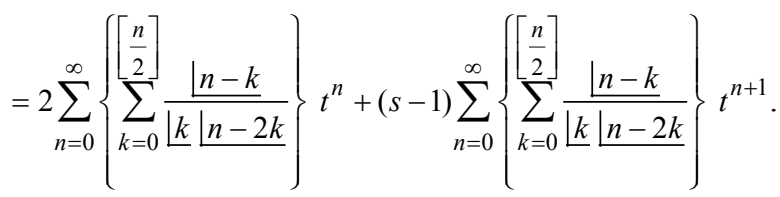

Equating the coefficient of $t^{n}$ we obtain

$$
M_{n}=2 \sum_{k=0}^{\left[\frac{n}{2}\right]}\left(\begin{array}{c}
n-k \\
k
\end{array}\right)+(s-1) \sum_{k=0}^{\left[\frac{n-1}{2}\right]}\left(\begin{array}{c}
n-k-1 \\
k
\end{array}\right) \text {. }
$$


For $s=1$ in above identity, explicit formulas can be obtained for Fibonacci sequence.

Theorem (3.2). (Sum of First $n$ terms) Sum of first $n$ terms of Generalized Fibonacci-Like sequence is

$$
\sum_{k=0}^{n} M_{k}=M_{n+2}-s-1 .
$$

Proof. By Binet's formula (2.4), we have

$$
\begin{aligned}
& \sum_{k=0}^{n} M_{k}=\sum_{k=0}^{n}\left[C_{1} \alpha^{k}+C_{2} \beta^{k}\right] \\
& =C_{1}\left[\frac{1-\alpha^{n+1}}{1-\alpha}\right]+C_{2}\left[\frac{1-\beta^{n+1}}{1-\beta}\right] \\
& =\frac{\left[\begin{array}{l}
\left(C_{1}+C_{2}\right)-\left(C_{1} \beta+C_{2} \alpha\right) \\
-\left(C_{1} \alpha^{n}+C_{1} \beta^{n}\right)+\alpha \beta\left(C_{1} \alpha^{n-1}+C_{1} \beta^{n-1}\right)
\end{array}\right]}{1-(\alpha+\beta)+\alpha \beta}
\end{aligned}
$$

Using subsequent results of Binet's formula, we get

$$
\sum_{k=0}^{n} M_{k}=M_{n+1}+M_{n}-s-1=M_{n+2}-s-1 . .
$$

Theorem (3.3). (Sum of First $n$ terms with odd indices): Sum of first $n$ terms (with odd indices) of Generalized Fibonacci-Like sequence is

$$
\sum_{k=1}^{n} M_{2 k-1}=M_{2 n+1}-M_{2 n-1}-2 .
$$

Proof. By Binet's formula (2.4), we have

$$
\begin{aligned}
\sum_{k=1}^{n} B_{2 k-1}=\sum_{k=1}^{n}\left[C_{1} \alpha^{2 k-1}+C_{2} \beta^{2 k-1}\right] \\
=C_{1}\left[\alpha+\alpha^{3}+\alpha^{5}+\ldots .+\alpha^{2 n-1}\right]+ \\
\quad C_{2}\left[\beta+\beta^{3}+\beta^{5}+\ldots .+\beta^{2 n-1}\right] \\
=-C_{1} \alpha\left[\frac{1-\alpha^{2 n}}{1-\alpha^{2}}\right]+C_{2} \beta\left[\frac{1-\beta^{2 n}}{1-\beta^{2}}\right] \\
=\frac{\left[\begin{array}{l}
\left(C_{1} \alpha^{2 n+1}+C_{2} \beta^{2 n+1}\right)-\left(C_{1} \alpha+C_{2} \beta\right) \\
+\alpha \beta\left(C_{1} \beta+C_{2} \alpha\right)-\alpha^{2} \beta^{2}\left(C_{1} \alpha^{2 n-1}+C_{1} \beta^{2 n-1}\right)
\end{array}\right] .}{\alpha^{2}+\beta^{2}-\alpha^{2} \beta^{2}-1} .
\end{aligned}
$$

Using subsequent results of Binet's formula, we get

$$
\sum_{k=1}^{n} M_{2 k-1}=M_{2 n+1}-M_{2 n-1}-2 .
$$

Theorem (3.4). (Sum of First $n$ terms with even indices) Sum of first $n$ terms (with even indices) of generalized Fibonacci-Like sequence is given by

$$
\sum_{k=0}^{n} M_{2 k}=M_{2 n}-M_{2 n-2}+1 .
$$

Proof. By Binet's formula (2.4), we have

$$
\begin{aligned}
& \sum_{k=0}^{n} M_{2 k}=\sum_{k=0}^{n}\left[C_{1} \alpha^{2 k}+C_{2} \beta^{2 k}\right] \\
& =-C_{1}\left[\frac{1-\alpha^{2 n}}{1-\alpha^{2}}\right]+C_{2}\left[\frac{1-\beta^{2 n}}{1-\beta^{2}}\right] \\
& =\frac{\left[\begin{array}{l}
\left(C_{1} \alpha^{2 n}+C_{2} \beta^{2 n}\right)-\left(C_{1}+C_{2}\right) \\
+\left(C_{1} \beta^{2}+C_{2} \alpha^{2}\right)-\alpha^{2} \beta^{2}\left(C_{1} \alpha^{2 n-2}+C_{1} \beta^{2 n-2}\right)
\end{array}\right]}{\alpha^{2}+\beta^{2}-\alpha^{2} \beta^{2}-1} .
\end{aligned}
$$

Using subsequent results of Binet's formula, we get

$$
\sum_{k=0}^{n} M_{2 k}=M_{2 n}-M_{2 n-2}+1 .
$$

Theorem (3.5). (Catalan's Identity) Let $M_{n}$ be the $n^{\text {th }}$ term of Generalized Fibonacci-Like sequence. Then

$$
\begin{aligned}
& M_{n}^{2}-M_{n+r} M_{n-r} \\
& =\frac{(-1)^{n-r}}{s^{2}-5}\left[(s+1) M_{r}-2 M_{r+1}\right]^{2}, n>r \geq 1 .
\end{aligned}
$$

Proof. By Binet's formula (2.4), we have

$$
\begin{aligned}
& M_{n}^{2}-M_{n+r} M_{n-r} \\
&=\left(C_{1} \alpha^{n}+C_{2} \beta^{n}\right)^{2} \\
&-\left(C_{1} \alpha^{n+r}+C_{2} \beta^{n+r}\right)\left(C_{1} \alpha^{n-r}+C_{2} \beta^{n-r}\right) \\
&= C_{1} C_{2}(\alpha \beta)^{n}\left(2-\alpha^{r} \beta^{-r}-\alpha^{-r} \beta^{r}\right) \\
&= C_{1} C_{2}(\alpha \beta)^{n-r}\left(2 \alpha^{r} \beta^{r}-\alpha^{2 r}-\beta^{2 r}\right) \\
&=-C_{1} C_{2}(\alpha \beta)^{n-r}\left(\alpha^{r}-\beta^{r}\right)^{2} .
\end{aligned}
$$

Using subsequent results of Binet's formula, we get

$$
M_{n}^{2}-M_{n+r} M_{n-r}=\left(s^{2}-5\right)(-1)^{n-r} \frac{\left(\alpha^{r}-\beta^{r}\right)^{2}}{(\alpha-\beta)^{2}} \text {. }
$$

Since $\frac{\alpha^{r}-\beta^{r}}{\alpha-\beta}=\frac{(s+1) M_{r}-2 M_{r+1}}{(s+1)^{2}-2(s+1)-4}$

$$
=\frac{(s+1) M_{r}-2 M_{r+1}}{s^{2}-5} \text {, }
$$

we obtain

$$
\begin{aligned}
& M_{n}^{2}-M_{n+r} M_{n-r} \\
& =\frac{(-1)^{n-r}}{s^{2}-5}\left[(s+1) M_{r}-2 M_{r+1}\right]^{2}, n>r \geq 1 .
\end{aligned}
$$

Corollary (3.5.1). (Cassini's Identity) Let $M_{n}$ be the $n^{\text {th }}$ term of Generalized Fibonacci-Like sequence. Then

$$
M_{n}^{2}-M_{n+1} M_{n-1}=(-1)^{n-1}\left(s^{2}-5\right), n \geq 1 .
$$

Taking $r=1$ in the Catalan's identity (3.5), the required identity is obtained.

Theorem (3.6). (d'Ocagne's Identity) Let $M_{n}$ be the $n^{\text {th }}$ term of generalized Fibonacci-Like sequence. Then 


$$
\begin{aligned}
& M_{m} M_{n+1}-M_{m+1} M_{n} \\
& =(-1)^{n}\left[(s+1) M_{m-n}-2 M_{m-n+1}\right], m>n \geq 0 .
\end{aligned}
$$

Proof. By Binet's formula (2.4), we have

$$
\begin{aligned}
& M_{m} M_{n+1}-M_{m+1} M_{n} \\
&=\left(C_{1} \alpha^{m}+C_{2} \beta^{m}\right)\left(C_{1} \alpha^{n+1}+C_{2} \beta^{n+1}\right) \\
&-\left(C_{1} \alpha^{m+1}+C_{2} \beta^{m+1}\right)\left(C_{1} \alpha^{n}+C_{2} \beta^{n}\right) \\
&= C_{1} C_{2}\left(\alpha^{m} \beta^{n+1}+\alpha^{n+1} \beta^{m}-\alpha^{n} \beta^{m+1}-\alpha^{m+1} \beta^{n}\right) \\
& M_{m} M_{n+1}-M_{m+1} M_{n} \\
&=C_{1} C_{2}(\alpha \beta)^{n}\left[\beta\left(\alpha^{m-n}-\beta^{m-n}\right)-\alpha\left(\alpha^{m-n}-\beta^{m-n}\right)\right] \\
&=- C_{1} C_{2}(\alpha \beta)^{n}(\alpha-\beta)\left(\alpha^{m-n}-\beta^{m-n}\right) .
\end{aligned}
$$

Using subsequent results of Binet's formula, we get

$M_{m} M_{n+1}-M_{m+1} M_{n}=(-1)^{n}\left(s^{2}-5\right) \frac{\left(\alpha^{m-n}-\beta^{m-n}\right)}{\alpha-\beta}$.

Since $\frac{\alpha^{m-n}-\beta^{m-n}}{\alpha-\beta}=\frac{(s+1) M_{m-n}-2 M_{m-n+1}}{\left(s^{2}-5\right)}$,

We get

$$
\begin{aligned}
& M_{m} M_{n+1}-M_{m+1} M_{n} \\
& =(-1)^{n}\left[(s+1) M_{m-n}-2 M_{m-n+1}\right], m>n \geq 0 .
\end{aligned}
$$

Theorem (3.7). (Generalized Identity) Let $M_{n}$ be the $n^{\text {th }}$ term of Generalized Fibonacci-Like sequence. Then

$$
\begin{aligned}
& M_{m} M_{n}-M_{m-r} M_{n+r} \\
& =\frac{(-1)^{m-r}}{\left(s^{2}-5\right)}\left\{(s+1) M_{r}-2 M_{r+1}\right\}\left\{\begin{array}{l}
(s+1) M_{n-m+r} \\
-2 M_{n-m+r+1}
\end{array}\right\},
\end{aligned}
$$

$n>m \geq r \geq 1$.

Proof. By Binet's formula (2.4), we have

$$
\begin{aligned}
& M_{m} M_{n}-M_{m-r} M_{n+r} \\
&=\left(C_{1} \alpha^{m}+C_{2} \beta^{m}\right)\left(C_{1} \alpha^{n}+C_{2} \beta^{n}\right) \\
&-\left(C_{1} \alpha^{m-r}+C_{2} \beta^{m-r}\right)\left(C_{1} \alpha^{n+r}+C_{2} \beta^{n+r}\right) \\
&= C_{1} C_{2}\left(\alpha^{r}-\beta^{r}\right)\left[\frac{\alpha^{m} \beta^{n}}{\alpha^{r}}-\frac{\alpha^{n} \beta^{m}}{\beta^{r}}\right] \\
&= C_{1} C_{2}(-1)^{-r}\left(\alpha^{r}-\beta^{r}\right)\left(\alpha^{m} \beta^{n+r}-\alpha^{n+r} \beta^{m}\right) \\
&= C_{1} C_{2}(-1)^{-r} \alpha^{m} \beta^{m}\left(\alpha^{r}-\beta^{r}\right)\left(\beta^{n-m+r}-\alpha^{n-m+r}\right) \\
&=-C_{1} C_{2}(-1)^{m-r}\left(\alpha^{r}-\beta^{r}\right)\left(\alpha^{n-m+r}-\beta^{n-m+r}\right) .
\end{aligned}
$$

Using subsequent results of Binet's formula, we get

$$
\begin{aligned}
& M_{m} M_{n}-M_{m-r} M_{n+r} \\
& =\frac{\left(s^{2}-5\right)}{(\alpha-\beta)^{2}}(-1)^{m-r}\left(\alpha^{r}-\beta^{r}\right)\left(\alpha^{n-m+r}-\beta^{n-m+r}\right) .
\end{aligned}
$$

Since $\frac{\alpha^{r}-\beta^{r}}{\alpha-\beta}=\frac{1}{s^{2}-5}\left\{(s+1) M_{r}-2 M_{r+1}\right\} \quad$ and

$$
\begin{aligned}
& \frac{\alpha^{n-m+r}-\beta^{n-m+r}}{\alpha-\beta} \\
& =\frac{1}{s^{2}-5}\left\{(s+1) M_{n-m+r}-2 M_{n-m+r+1}\right\},
\end{aligned}
$$

we obtain

$M_{m} M_{n}-M_{m-r} M_{n+r}$

$=\frac{(-1)^{m-r}}{\left(s^{2}-5\right)}\left\{(s+1) M_{r}-2 M_{r+1}\right\}\left\{\begin{array}{l}(s+1) M_{n-m+r} \\ -2 M_{n-m+r+1}\end{array}\right\}$,

$n>m \geq r \geq 1$.

The identity (3.8) provides Catalan's, Cassini's and d'Ocagne's and other identities:

(i) If $\mathrm{m}=\mathrm{n}$, the Catalan's identity (3.5) is obtained.

(ii) If $\mathrm{m}=\mathrm{n}$ and $r=1$ in identity (3.8), the Cassini's identity (5.1) is obtained.

(iii) $\mathrm{n}=\mathrm{m}, \mathrm{m}=n+1$ and $r=1$ in identity (3.8), the d'Ocagne's identity (3.6) is obtained.

\section{Determinant Identities}

There is a long tradition of using matrices and determinants to study Fibonacci numbers. Problems on determinants of Fibonacci sequence and Lucas sequence are appeared in various issues of Fibonacci Quarterly. T. Koshy [13] explained two chapters on the use of matrices and determinants. Many determinant identities of generalized Fibonacci sequence are discussed in [4], [6] and [11]. In this section some determinant identities of Generalized Fibonacci-Like sequence are presented. Entries of determinants are satisfying the recurrence relation of Generalized Fibonacci-Like sequence and other sequences.

Theorem(4.1). For any integers $n \geq 0$, prove that

$$
\left|\begin{array}{lll}
M_{n+1} & \mathrm{M}_{n+2} & \mathrm{M}_{n+3} \\
M_{n+4} & \mathrm{M}_{n+5} & \mathrm{M}_{n+6} \\
M_{n+7} & \mathrm{M}_{n+8} & \mathrm{M}_{n+9}
\end{array}\right|=0
$$

Proof.

$$
\text { Let } \Delta=\left|\begin{array}{lll}
M_{n+1} & \mathrm{M}_{n+2} & \mathrm{M}_{n+3} \\
M_{n+4} & \mathrm{M}_{n+5} & \mathrm{M}_{n+6} \\
M_{n+7} & \mathrm{M}_{n+8} & \mathrm{M}_{n+9}
\end{array}\right|
$$

Applying $C_{1} \rightarrow C_{1}+C_{2}$, we get

$$
\text { Let } \Delta=\left|\begin{array}{lll}
M_{n+3} & \mathrm{M}_{n+2} & \mathrm{M}_{n+3} \\
M_{n+6} & \mathrm{M}_{n+5} & \mathrm{M}_{n+6} \\
M_{n+9} & \mathrm{M}_{n+8} & \mathrm{M}_{n+9}
\end{array}\right|
$$

Since two columns are identical, thus we obtained required result.

Theorem (4.2). For any integer $n \geq 0$, prove that

$$
\left|\begin{array}{ccc}
M_{n}-M_{n+1} & \mathrm{M}_{n+1}-M_{n+2} & \mathrm{M}_{n+2}-M_{n} \\
M_{n+1}-M_{n+2} & \mathrm{M}_{n+2}-M_{n} & \mathrm{M}_{n}-M_{n+1} \\
M_{n+2}-M_{n} & \mathrm{M}_{n}-M_{n+1} & \mathrm{M}_{n+1}-M_{n+2}
\end{array}\right|=0 .
$$

\section{Proof.}


Let $\Delta=\left|\begin{array}{lll}M_{n}-M_{n+1} & \mathrm{M}_{n+1}-M_{n+2} & \mathrm{M}_{n+2}-M_{n} \\ M_{n+1}-M_{n+2} & \mathrm{M}_{n+2}-M_{n} & \mathrm{M}_{n}-M_{n+1} \\ M_{n+2}-M_{n} & \mathrm{M}_{n}-M_{n+1} & \mathrm{M}_{n+1}-M_{n+2}\end{array}\right|$.

By applying $C_{1} \rightarrow \mathrm{C}_{1}+C_{2}+C_{3}$ and expanding along first row, we obtained required result.

Theorem (4.3). For any integer $n \geq 0$, prove that

$$
\left|\begin{array}{cll}
1 & 1 & 1 \\
\mathrm{M}_{n} & \mathrm{M}_{n+1} & \mathrm{M}_{n+2} \\
M_{n+1}+M_{n+2} & \mathrm{M}_{n}+M_{n+2} & \mathrm{M}_{n}+M_{n+1}
\end{array}\right|=0 .
$$

\section{Proof.}

$$
\text { Let } \Delta=\left|\begin{array}{ccc}
1 & 1 & 1 \\
\mathrm{M}_{n} & \mathrm{M}_{n+1} & \mathrm{M}_{n+2} \\
M_{n+1}+M_{n+2} & \mathrm{M}_{n}+M_{n+2} & \mathrm{M}_{n}+M_{n+1}
\end{array}\right| \text {. }
$$

Applying $R_{3} \rightarrow R_{3}+R_{2}$, we get

$$
\Delta=\left|\begin{array}{lll}
1 & 1 & 1 \\
M_{n} & \mathrm{M}_{n+1} & \mathrm{M}_{n+2} \\
2 M_{n+2} & 2 M_{n+2} & 2 M_{n+2}
\end{array}\right| .
$$

Taking common out $2 M_{n+2}$ from third row,

$$
\Delta=2 M_{n+2}\left|\begin{array}{ccc}
1 & 1 & 1 \\
M_{n} & \mathrm{M}_{n+1} & \mathrm{M}_{n+2} \\
1 & 1 & 1
\end{array}\right| .
$$

Since two rows are identical, thus we obtained required result.

Theorem (4.4). For any integer $n \geq 0$, prove that

$$
\begin{aligned}
& \left|\begin{array}{ccc}
\mathrm{M}_{n} & \mathrm{M}_{n}+M_{n+1} & \mathrm{M}_{n}+M_{n+1}+M_{n+2} \\
2 M_{n} & 2 \mathrm{M}_{n}+3 M_{n+1} & 2 \mathrm{M}_{n}+3 M_{n+1}+4 M_{n+2} \\
3 \mathrm{M}_{n} & 3 \mathrm{M}_{n}+6 M_{n+1} & 3 \mathrm{M}_{n}+6 M_{n+1}+12 M_{n+2}
\end{array}\right|(4.4) \\
& =3 M_{n} M_{n+1} M_{n+2} .
\end{aligned}
$$

Proof. Let

$\Delta=\left|\begin{array}{ccc}\mathrm{M}_{n} & \mathrm{M}_{n}+M_{n+1} & \mathrm{M}_{n}+M_{n+1}+M_{n+2} \\ 2 M_{n} & 2 \mathrm{M}_{n}+3 M_{n+1} & 2 \mathrm{M}_{n}+3 M_{n+1}+4 M_{n+2} \\ 3 \mathrm{M}_{n} & 3 \mathrm{M}_{n}+6 M_{n+1} & 3 \mathrm{M}_{n}+6 M_{n+1}+12 M_{n+2}\end{array}\right|$.

Applying $R_{2} \rightarrow R_{2}-2 R_{1}, R_{3} \rightarrow R_{3}-3 R_{1}$, we get

$$
\Delta=\left|\begin{array}{ccc}
M_{n} & \mathrm{M}_{n}+\mathrm{M}_{n+1} & \mathrm{M}_{n}+\mathrm{M}_{n+1}+M_{n+2} \\
0 & \mathrm{M}_{n+1} & \mathrm{M}_{n+1}+2 \mathrm{M}_{n+2} \\
0 & 3 \mathrm{M}_{n+1} & 3 \mathrm{M}_{n+1}+9 M_{n+2}
\end{array}\right| .
$$

Applying $R_{3} \rightarrow R_{3}-3 R_{2}$ and expanding along first row, we obtained required result.

Theorem (4.5). For any integer $n \geq 0$, prove that

$$
\begin{aligned}
& \left|\begin{array}{ccc}
0 & \mathrm{M}_{n} M_{n+1}^{2} & \mathrm{M}_{n} M_{n+2}^{2} \\
M_{n}^{2} M_{n+1} & 0 & \mathrm{M}_{n+1} M_{n+2}^{2} \\
M_{n}^{2} M_{n+2} & \mathrm{M}_{n+2} M_{n+1}^{2} & 0
\end{array}\right| \\
& =2 M_{n}^{3} M_{n+1}^{3} M_{n+2}^{3} .
\end{aligned}
$$

Proof. Let

$$
\Delta=\left|\begin{array}{ccc}
0 & \mathrm{M}_{n} M_{n+1}^{2} & \mathrm{M}_{n} M_{n+2}^{2} \\
M_{n}^{2} M_{n+1} & 0 & \mathrm{M}_{n+1} M_{n+2}^{2} \\
M_{n}^{2} M_{n+2} & \mathrm{M}_{n+2} M_{n+1}^{2} & 0
\end{array}\right| .
$$

Taking common out $M_{n}^{2}, \mathrm{M}_{n+1}^{2}, \mathrm{M}_{n+2}^{2}$ from $C_{1}, C_{2}, C_{3}$ respectively, we get

$$
\Delta=\mathrm{M}_{n}^{2} M_{n+1}^{2} M_{n+2}^{2}\left|\begin{array}{ccc}
0 & \mathrm{M}_{n} & \mathrm{M}_{n} \\
M_{n+1} & 0 & \mathrm{M}_{n+1} \\
M_{n+2} & \mathrm{M}_{n+2} & 0
\end{array}\right| .
$$

Taking common out $M_{n}, \mathrm{M}_{n+1}, \mathrm{M}_{n+2}$ from $R_{1}, \mathrm{R}_{2}, \mathrm{R}_{3}$ respectively and expanding along first row, we obtained required result.

Theorem (4.6). For any integer $n \geq 0$, prove that

$$
\left|\begin{array}{ccc}
M_{\mathrm{n}} & \mathrm{F}_{\mathrm{n}} & 1 \\
M_{\mathrm{n}+1} & \mathrm{~F}_{\mathrm{n}+1} & 1 \\
M_{\mathrm{n}+2} & \mathrm{~F}_{\mathrm{n}+2} & 1
\end{array}\right|=\left[F_{n} M_{n+1}-M_{n} F_{n+1}\right] .
$$

Proof: Let $\Delta=\left|\begin{array}{ccc}M_{\mathrm{n}} & \mathrm{F}_{\mathrm{n}} & 1 \\ M_{\mathrm{n}+1} & \mathrm{~F}_{\mathrm{n}+1} & 1 \\ M_{\mathrm{n}+2} & \mathrm{~F}_{\mathrm{n}+2} & 1\end{array}\right|$.

Assume $M_{n}=a, \mathrm{M}_{n+1}=b, \mathrm{~F}_{n}=\mathrm{p}, \mathrm{F}_{n+1}=\mathrm{q}$ then $\mathrm{M}_{n+2}=a+b$ and $\mathrm{F}_{n+2}=\mathrm{p}+\mathrm{q}$

Now substituting the above values in determinant, we get

$$
\Delta=\left|\begin{array}{ccc}
a & p & 1 \\
b & q & 1 \\
a+b & p+q & 1
\end{array}\right|
$$

Applying $R_{1} \rightarrow R_{1}-R_{2}$

$$
\Delta=\left|\begin{array}{ccc}
a-b & p-q & 0 \\
b & q & 1 \\
a+b & p+q & 1
\end{array}\right|
$$

Applying $R_{2} \rightarrow R_{2}-R_{3}$

$$
\Delta=\left|\begin{array}{ccc}
a-b & p-q & 0 \\
-a & -p & 0 \\
a+b & p+q & 1
\end{array}\right|=(p b-a q) .
$$

Substituting the values of $a, b, p$ and $q$, we get required result.

Similarly following identities can be derived:

Theorem (4.7). For any integer $n \geq 0$, prove that

$$
\left|\begin{array}{ccc}
M_{n} & M_{n+1} & M_{n+2} \\
M_{n+2} & M_{n} & M_{n+1} \\
M_{n+1} & M_{n+2} & M_{n}
\end{array}\right|=2\left(M_{n}^{3}+M_{n+1}^{3}\right) .
$$

Theorem (4.8). For any integer $n \geq 0$, prove that

$$
\left|\begin{array}{ccc}
M_{n} & L_{n} & 1 \\
M_{n+1} & L_{n+1} & 1 \\
M_{n+2} & L_{n+2} & 1
\end{array}\right|=2\left(L_{n} M_{n+1}-M_{n} L_{n+1}\right) .
$$


Theorem (4.9). For any integer $n \geq 0$, prove that

$$
\left|\begin{array}{ccc}
M_{n}+M_{n+1} & M_{n+1}+M_{n+2} & M_{n+2}+M_{n} \\
M_{n+2} & M_{n} & M_{n+1} \\
1 & 1 & 1
\end{array}\right|=0 .
$$

Theorem 4.(10). For any integer $n \geq 0$, prove that

$$
\begin{aligned}
& \left|\begin{array}{ccc}
1+M_{n} & M_{n+1} & M_{n+2} \\
M_{n} & 1+M_{n+1} & M_{n+2} \\
M_{n} & M_{n+1} & 1+M_{n+2}
\end{array}\right| \\
& =1+M_{n}+M_{n+1}+M_{n+2} .
\end{aligned}
$$

\section{Acknowledgement}

We would like to thank the anonymous referees for numerous helpful suggestions.

\section{References}

[1] A. F. Horadam: A Generalized Fibonacci sequence, American Mathematical Monthly, Vol. 68. (5), 1961, 455-459.

[2] A. F. Horadam: Basic Properties of a Certain Generalized Sequence of Numbers, The Fibonacci Quarterly, Vol. 3 (3), 1965, 161-176.
[3] A. T. Benjamin and D. Walton, Counting on Chebyshev polynomials, Math. Mag. 82, 2009, 117-126.

[4] B. Singh, O. Sikhwal and S. Bhatnagar: Fibonacci-Like Sequence and its Properties, Int. J. Contemp. Math. Sciences, Vol. 5 (18), 2010, 859-868.

[5] B. Singh, S. Bhatnagar and O. Sikhwal: Fibonacci-Like Polynomials and some Identities, International Journal of Advanced Mathematical Sciences, 1(3),(2013),152-157.

[6] B. Singh, S. Bhatnagar and O. Sikhwal: Fibonacci-Like Sequence, International Journal of Advanced Mathematical Sciences, 1 (3) (2013), 145-151.

[7] B. Singh, S. Bhatnagar and O. Sikhwal: Generalized Identties of Companion Fibonacci-Like Sequences, Global Journal of Mathematical Analysis, 1 (3) 2013, 104-109.

[8] D. V. Jaiswal: On a Generalized Fibonacci sequence, Labdev J. Sci. Tech. Part A 7, 1969, 67-71.

[9] M. Edson and O. Yayenie: A New Generalization of Fibonacci sequence and Extended Binet's Formula, Integers Vol. 9, 2009, 639-654.

[10] M. E. Waddill and L. Sacks: Another Generalized Fibonacci sequence, The Fibonacci Quarterly, Vol. 5 (3), 1967, 209-222.

[11] M. Singh, Y. Gupta, O. Sikhwal, Generalized Fibonacci-Lucas Sequences its Properties, Global Journal of Mathematical Analysis, 2(3), 2014, 160-168.

[12] O. Sikhwal, Generalization of Fibonacci Sequence: An Intriguing Sequence, Lap Lambert Academic Publishing GmbH \& Co. KG, Germany (2012).

[13] S. Falcon and A. Plaza: On the Fibonacci $K$ - Numbers, Chaos, Solutions \& Fractals, Vol. 32 (5), 2007, 1615-1624.

[14] S. Vajda, Fibonacci \& Lucas Numbers and the Golden Section, Theory and Applications, Ellis Horwood Ltd., Chichester, 1989.

[15] T. Koshy, Fibonacci and Lucas Numbers with Applications, Wiley- Interscience Publication, New York (2001). 